\title{
Dietary flavanol intervention lowers the levels of endothelial microparticles in coronary artery disease patients
}

\author{
Patrick Horn ${ }^{1}$, Nicolas Amabile ${ }^{2}$, Franca S. Angeli ${ }^{2}$, Roberto Sansone ${ }^{1}$, Berthold Stegemann ${ }^{1}$, \\ Malte Kelm ${ }^{1}$, Matthew L. Springer ${ }^{2,3,4}$, Yerem Yeghiazarians ${ }^{2,3,4}$, Hagen Schroeter ${ }^{5,6}$ \\ and Christian Heiss ${ }^{1,2 *}$ \\ ${ }^{1}$ Division of Cardiology, Pulmonology, and Vascular Medicine, Medical Faculty, University Duesseldorf, Moorenstrasse 5, \\ Düsseldorf D-40235, Germany \\ ${ }^{2}$ Division of Cardiology, University of California San Francisco, San Francisco, CA, USA \\ ${ }^{3}$ Cardiovascular Research Institute, University of California San Francisco, San Francisco, CA, USA \\ ${ }^{4}$ Eli and Edythe Broad Center of Regeneration Medicine and Stem Cell Research, University of California San Francisco, \\ San Francisco, CA, USA \\ ${ }^{5}$ Nutrition Department, University of California Davis, Davis, CA, USA \\ ${ }^{6}$ Mars, Inc., McLean, VA, USA \\ (Submitted 21 May 2013 - Final revision received 26 September 2013 - Accepted 9 October 2013 - First published online 29 November 2013 )
}

\section{Abstract}

Current evidence suggests that regenerative $v$. degenerative endothelial responses can be integrated in a clinical endothelial phenotype, reflecting the net result between damage from risk factors and endogenous repair capacity. We have previously shown that a cocoa flavanol (CF) intervention can improve endothelial function and increase the regenerative capacity of the endothelium by mobilising circulating angiogenic cells in patients with coronary artery disease (CAD). The aim of the present study was to investigate whether CF can lower the levels of circulating endothelial microparticles (EMP), markers of endothelial integrity, along with improvements in endothelial function. The levels of EMP in the frozen plasma samples of CAD patients were measured along with endothelial function (flow-mediated vasodilation, FMD); $n$ 16, FMD data published previously), and these data were compared with those of young ( $n$ 12) and age-matched $(n$ 12) healthy control subjects. The CAD patients exhibited significantly increased levels of EMP along with impaired FMD when compared with the healthy control subjects. The levels of CD $144^{+}$and $\mathrm{CD} 31^{+} / 41^{-}$EMP were inversely correlated with FMD $(r-0.67, P=0.01$ and $r-0.59, P=0 \cdot 01$, respectively). In these CAD patients, the levels of EMP were measured after they had consumed a drink containing $375 \mathrm{mg}$ of CF (high-CF intervention, HiFI) or $9 \mathrm{mg}$ of CF (macro- and micronutrient-matched low-CF control, LoFl) twice daily over a 30-d period in a randomised, double-blind, cross-over study. After 1 month of HiFI, the levels of CD31 $/ 41^{-}$ and $\mathrm{CD} 144^{+}$EMP decreased ( -25 and $-23 \%$, respectively), but not after LoFl. Our data show that flavanols lower the levels of EMP along with higher endothelial function, lending evidence to the novel concept that flavanols may improve endothelial integrity.

Key words: Cocoa flavanols: Endothelial integrity: Endothelial function: Endothelial microparticles

The vascular endothelium has emerged as a dynamic organ that responds to environmental factors. According to the response to injury theory, mechanical injury and exposure to cardiovascular risk factors impair the regulatory functions of the endothelium and contribute to a proinflammatory phenotype, senescence and apoptosis ${ }^{(1)}$. As a consequence, the endothelial integrity can be disrupted with endothelial cells or parts of these cells being detached and released into the circulation ${ }^{(2)}$. Therefore, endothelial microparticles (EMP) can be viewed as circulating markers of a compromised endothelial integrity that are released from activated and apoptotic endothelial cells ${ }^{(3,4)}$. These EMP are membrane particles of less than $1 \mu \mathrm{m}$ in diameter that carry endothelial surface markers ${ }^{(3-5)}$ and enzymes including endothelial NO synthase ${ }^{(6)}$. The levels of circulating EMP increase in plasma early in atherosclerotic processes and correlate with the degree of endothelial dysfunction ${ }^{(7)}$ and have been established as prognostic biomarkers that predict adverse CVD outcome ${ }^{(8-10)}$.

Abbreviations: CAC, circulating angiogenic cells; CAD, coronary artery disease; CF, cocoa flavanol; EMP, endothelial microparticles; FMD, flow-mediated vasodilation; HiFI, high-cocoa flavanol intervention; LoFl, low-cocoa flavanol control; MP, microparticles.

*Corresponding author: C. Heiss, fax +49211 8118812, email christian.heiss@med.uni-duesseldorf.de 
Cardiovascular risk factors may trigger the release of $\operatorname{EMP}^{(5,11)}$. For instance, smoking, as well as second-hand smoking, forced physical inactivity and increased blood pressure have been reported to be associated with increases in the levels of circulating EMP in healthy subjects ${ }^{(12-14)}$. Increases in the plasma levels of EMP have also been observed after consumption of high-fat meals with augmented circulating levels of modified LDL and TAG ${ }^{(15)}$. Conversely, Mediterranean diet has been shown to lower the levels of circulating EMP in healthy elderly subjects, indicating that diet can improve endothelial integrity ${ }^{(16-18)}$.

Dietary flavanols have emerged as promising protectants of cardiovascular function and health ${ }^{(19)}$. In the context of human nutrition, major flavanol sources in the diet include tea, wine, cocoa products and, more seasonally, various fruit and vegetables. Especially, high concentrations of flavanols can be present in $\mathrm{COcoa}^{(20)}$. It has been shown that acute and chronic ingestion of cocoa flavanols (CF) can improve endothelial function in healthy subjects and patients with diabetes and coronary artery disease $(\mathrm{CAD})^{(19)}$. We have previously shown that $\mathrm{CF}$ may not only directly improve endothelial function ( $4 \cdot 5-8 \cdot 4 \%$ ), but also enhance endothelial regenerative capacity by mobilising circulating angiogenic cells (CAC) in patients with $\mathrm{CAD}^{(21)}$. Whether a CF intervention improves or even restores endothelial integrity is unknown.

Therefore, the aim of the present study was to investigate whether the CF-related improvement in endothelial function that we had observed in CAD patients ${ }^{(21)}$ was accompanied by a decrease in the levels of circulating EMP, markers of endothelial integrity, in plasma obtained from the same subjects. To put the absolute values of EMP in the context of the cardiovascular health continuum, we report crosssectional data obtained from healthy young and age-matched control subjects.

\section{Methods}

\section{Study design}

We analysed microparticles (MP) in frozen plasma samples that were obtained from CAD patients and stored during our previously published clinical study showing that a high-CF drink (HiFI) is capable of improving endothelial function and mobilise $\mathrm{CAC}$ in these patients ${ }^{(21)}$. CAD patients were characterised by chronic stable angina, were on optimal medical therapy and had achieved treatment goals according to the current American Heart Association/American College of Cardiology (AHA/ACC) guidelines as indicated by baseline characteristics and medication (Table 1). The CAD patients ( $n$ 16) were given a HiFI (375 mg of CF) or a nutrientmatched low-CF drink (LoFl, $9 \mathrm{mg}$ of CF) twice daily over a 30-d period, using a randomised, double-masked, cross-over study design with 1 week of wash-out between the interventions (for a detailed published protocol, see Heiss et $a l{ }^{(21)}$ ). Measurements and blood draws were carried out before the initiation of each intervention (day 0, 'pre') and the day after the completion of each intervention (day 30, 'post'). Preand post-intervention plasma samples were collected after an overnight fast and following $30 \mathrm{~min}$ of supine rest. All individual medications and treatment paradigms remained unaltered throughout the study period. Randomisation and

Table 1. Baseline characteristics of coronary artery disease (CAD) patients and comparator healthy controls

\begin{tabular}{|c|c|c|c|c|c|c|c|}
\hline & \multicolumn{2}{|c|}{ CAD patients } & \multicolumn{2}{|c|}{$\begin{array}{l}\text { Healthy young } \\
\text { controls }\end{array}$} & \multicolumn{2}{|c|}{$\begin{array}{l}\text { Healthy old } \\
\text { controls }\end{array}$} & \multirow[b]{2}{*}{$P$} \\
\hline & Mean & SD & Mean & SD & Mean & SD & \\
\hline Subjects $(n)$ & \multicolumn{2}{|c|}{16} & \multicolumn{2}{|c|}{12} & \multicolumn{2}{|c|}{12} & \\
\hline Male & \multicolumn{2}{|c|}{13} & \multicolumn{2}{|c|}{8} & \multicolumn{2}{|c|}{7} & \\
\hline Female & \multicolumn{2}{|c|}{3} & \multicolumn{2}{|c|}{4} & \multicolumn{2}{|c|}{5} & \\
\hline Age (years) & 64 & 3 & $27^{\star}$ & 4 & 60 & 10 & $<0.001$ \\
\hline $\mathrm{BMI}\left(\mathrm{kg} / \mathrm{m}^{2}\right)$ & $28 \cdot 8$ & 1.8 & $23 \cdot 1^{*}$ & 0.3 & 27.5 & 0.7 & $<0.001$ \\
\hline Diabetes mellitus (\%) & \multicolumn{2}{|c|}{38} & \multicolumn{2}{|c|}{0} & \multicolumn{2}{|c|}{0} & \\
\hline Hypertension (\%) & \multicolumn{2}{|c|}{88} & & & \multicolumn{2}{|c|}{0} & \\
\hline Hyperlipidaemia (\%) & \multicolumn{2}{|c|}{94} & & & \multicolumn{2}{|c|}{0} & \\
\hline Prior smoking (\%) & \multirow{2}{*}{\multicolumn{2}{|c|}{$\begin{array}{c}63 \\
100\end{array}$}} & & & \multicolumn{2}{|c|}{0} & \\
\hline ACE inhibitor/angiotensin receptor blocker (\%) & & & & & \multicolumn{2}{|c|}{0} & \\
\hline Aspirin (\%) & \multicolumn{2}{|c|}{94} & & & \multicolumn{2}{|c|}{0} & \\
\hline$\beta$-Blocker (\%) & \multicolumn{2}{|c|}{88} & & & & & \\
\hline Statin (\%) & \multicolumn{2}{|c|}{94} & \multicolumn{2}{|c|}{0} & \multicolumn{2}{|c|}{0} & \\
\hline Heart rate (beats per min) & 60 & 2 & 62 & 6 & 58 & 6 & 0.890 \\
\hline Systolic blood pressure $(\mathrm{mmHg})$ & 132 & 2 & $122^{*}$ & 3 & 130 & 12 & 0.015 \\
\hline Diastolic blood pressure (mmHg) & 75 & 2 & $61^{\star}$ & 3 & 77 & 12 & $<0.001$ \\
\hline Total cholesterol (mg/l) & 1470 & 80 & 1490 & 670 & $1920^{*}$ & 70 & $<0.001$ \\
\hline LDL-cholesterol (mg/l) & 800 & 70 & 890 & 80 & $1270^{*}$ & 60 & $<0.001$ \\
\hline HDL-cholesterol (mg/l) & 460 & 30 & $620^{\star}$ & 50 & 600 & 20 & 0.034 \\
\hline TAG (mg/l) & 1070 & 140 & 1080 & 110 & 1150 & 390 & 0.644 \\
\hline Fasting glucose $(\mathrm{mg} / \mathrm{l})$ & 1040 & 90 & 860 & 70 & 960 & 110 & 0.125 \\
\hline Leucocytes $(\mu \mathrm{l})$ & 5900 & 40 & 6500 & 600 & 5460 & 300 & 0.621 \\
\hline Platelets $(\mu \mathrm{l})$ & 217 & 15 & 230 & 15 & 229 & 15 & 0.115 \\
\hline hs-CRP (mg/l) & 1.8 & 0.3 & 0.5 & 0.3 & 0.5 & 0.3 & 0.023 \\
\hline
\end{tabular}

ACE, angiotensin-converting enzyme; hs-CRP, high-sensitivity C-reactive protein.

${ }^{\star}$ Mean value was significantly different from that of the CAD patients $(P<0.05)$. 
drink dispensations were carried out by the Department of Pharmacy, University of California, San Francisco, USA. The patients and investigators were blinded throughout the study with regard to the $\mathrm{CF}$ content of the test drinks.

Inclusion of the patient group was based on a diagnosis of CAD as defined by $>70 \%$ stenosis of at least one coronary artery and the indication of medical therapy. Optimal medical therapy was defined as treatment with angiotensin-converting enzyme (ACE) inhibitors and/or angiotensin receptor blockers, $\beta$-blockers, aspirin and a statin, unless contraindicated or not tolerated according to the current AHA/ACC secondary prevention guidelines ${ }^{(22)}$. General exclusion criteria were the presence of heart rhythms other than sinus rhythm, clinical diagnosis of heart failure (New York Heart Association (NYHA) III and IV), recent or current inflammatory condition, terminal renal insufficiency, active malignancies within the last year, documented malcompliance with medication, the current use of vitamin and herbal supplementation, active smoking ( $>1$ cigarette/d), allergies to chocolate, cocoa, flavanols, or related products, and lactose and caffeine intolerance.

In order to put the MP levels of CAD patients as determined before and after CF intervention in context with healthy subjects, we determined MP levels and endothelial function in healthy young and older subjects age-matched to CAD patients (Table $1)$. The healthy control groups were defined as young ( $<35$ years) and older subjects matched to the age of CAD patients. Cardiovascular health was defined by the absence of CVD (CAD, peripheral arterial disease and cerebrovascular disease) and absence of cardiovascular risk factors including hypertension (blood pressure $>140 />90 \mathrm{mmHg}$ ), hypercholesterolaemia (total cholesterol levels $>2000 \mathrm{mg} / \mathrm{l}$ ) and smoking. Furthermore, general exclusion criteria outlined above were applied. The present study was conducted according to the guidelines laid down in the Declaration of Helsinki, and all procedures involving human subjects were approved by the UCSF Committee on Human Research (Clinicaltrials.gov: NCT00553774). Written informed consent was witnessed and formally recorded.

\section{Flavanol-containing test materials}

The test drinks were supplied as dry beverage mixes by Mars Incorporated. All the drinks were standardised for their CF content and profile and closely matched for equal macro- and micronutrient content, energy load, and theobromine and caffeine levels (Table 2). All the drinks were similar in taste and supplied in individual, opaque sachets containing $6 \mathrm{~g}$ of beverage mix. A sachet contained $9 \mathrm{mg}$ (LoFl) or $375 \mathrm{mg}$ (HiFI) of CF. The amount of CF referenced here is defined as the sum of all monomeric flavanols and their oligomeric derivatives (dimers to decamers, i.e. 2-10 monomeric subunits).

\section{Measurement of flow-mediated dilation}

The endothelial function of the CAD patients as well as of the healthy subjects was assessed by measuring the flowmediated vasodilation (FMD) of the brachial artery by ultrasound (Sonosite Micromaxx, SonoSite, Inc.) by the same
Table 2. Composition of flavanol-containing test materials

\begin{tabular}{lcc}
\hline & LoFI & HiFI \\
\hline CF (mg) & 9 & 375 \\
Monomers (mg) & 3 & 65 \\
$\quad$ Epicatechin (mg) & 1 & 59 \\
Catechin (mg) & 2 & 6 \\
Dimers (mg) & 2 & 53 \\
Trimers-decamers (mg) & 3 & 258 \\
Theobromine (mg) & 96 & 93 \\
Caffeine (mg) & 9 & 11 \\
Energy (kcal) & 25 & 25 \\
Energy (kJ) & 105 & 105 \\
Fat (g) & 0.3 & 0.4 \\
Carbohydrate (g) & 4.6 & 4.3 \\
Protein (g) & 0.7 & 0.7 \\
\hline
\end{tabular}

LoFI, low-flavanol control; HiFI, high-CF intervention; CF, cocoa flavanols.

investigator in combination with an automated analysis system (Brachial Analyzer, Medical Imaging Applications) in a $21^{\circ} \mathrm{C}$ temperature-controlled room as described previously $^{(21)}$. The image and flow analyses were carried out offline from recorded loops with an automated system (Brachial Analyzer 5, Medical Imaging Applications). All the diameter readings were taken at diastole, and flow velocity represents the mean angle-corrected Doppler flow velocity. Vasodilation results are presented as percentage change:

diameter $_{\text {post-ischaemia }}-$ diameter $_{\text {baseline }} /$ diameter $_{\text {baseline }} \times 100$.

\section{Blood collection and preparation of platelet-free plasma}

Citrated blood $(6 \mathrm{ml})$ was drawn from the cubital vein. All the samples were processed within $2 \mathrm{~h}$ of blood draw. Platelet-rich plasma was obtained by centrifugation at $300 \boldsymbol{g}$ over $15 \mathrm{~min}$ at room temperature. Platelet-free plasma was obtained by two successive centrifugations of the supernatants at $10000 \boldsymbol{g}$ for $5 \mathrm{~min}$ at room temperature. Platelet-free plasma obtained by this sequential centrifugation contains MP but not platelets, as shown by flow cytometry and fluorescence-based laser-scanning microscopy $(\mathrm{Carl} \text { Zeiss Jena } \mathrm{GmbH})^{(6)}$. The samples were stored at $-80^{\circ} \mathrm{C}$. The samples of the CAD patients and control subjects were handled the same way regarding sample preparation, storage and performance of flow cytometry.

\section{Characterisation of microparticles subpopulations by flow cytometry}

MP subpopulations were discriminated by flow cytometry according to the expression of established surface antigens ${ }^{(6)}$. The samples were incubated for $30 \mathrm{~min}$ with fluorochromelabelled antibodies or matching isotype controls and analysed in a Canto II flow cytometer (Becton Dickinson). The events were discriminated by $1.0 \mu \mathrm{m}$ microbead standards (Polyscience, Inc.). Platelet-derived MP were defined as $\mathrm{CD}_{4} 1^{+}$ MP, whereas EMP subpopulations were defined as CD144 $4^{+}$ or $\mathrm{CD}^{+} / \mathrm{CD}^{-} 1^{-}$. Phycoerythrin-cyanin 5 (PC5)-conjugated mouse anti-human CD41 and phycoerythrin (PE)-conjugated 
mouse anti-human CD144 antibodies were obtained from Beckman Coulter. PE-conjugated mouse anti-human CD31 was obtained from Becton Dickinson. The total number of MP was quantified with flow count calibrator beads (Beckman Coulter; $20 \mu \mathrm{l}$ ). Unless specified otherwise, chemicals were purchased from Sigma Aldrich.

\section{Measurement of procoagulant activity of microparticles}

The procoagulant activity of MP was assessed using a two-step amidolytic assay based on the ability of the tissue factor to generate Factor Xa in the presence of an excess of Factors VII and X. The supernatant of platelet-free plasma (PFP) was centrifuged at $14000 \mathrm{~g}$ for $90 \mathrm{~min}$. The supernatant was discarded, and the MP pellet was gently resuspended in $1 \mathrm{ml}$ of filtered $0 \cdot 1 \% \mathrm{PBS} /$ bovine serum albumin. This MP suspension was again centrifuged for $90 \mathrm{~min}$ at $14000 \mathrm{~g}$ and $4^{\circ} \mathrm{C}$, and the supernatant was removed. After the second washing, the MP pellet was resuspended in $70 \mu \mathrm{l}$ of $0.1 \% \mathrm{PBS} /$ bovine serum albumin buffer. In a ninety-six-well microtitre plate, $25 \mu \mathrm{l}$ of MP were incubated with $50 \mu \mathrm{l}$ of the mixture containing $0.5 \mu \mathrm{l}$ of human factor VII (Kordia; initial concentration $46 \mu \mathrm{g} / \mathrm{ml}$ ), $1 \mu \mathrm{l}$ of human factor X (Kordia; $1 \mathrm{mg} / \mathrm{ml}$ ) and $50 \mu \mathrm{l}$ of Tris-buffered saline (100 mm-Tris- $\mathrm{HCl}, 240 \mathrm{~mm}-\mathrm{NaCl}$, (a)

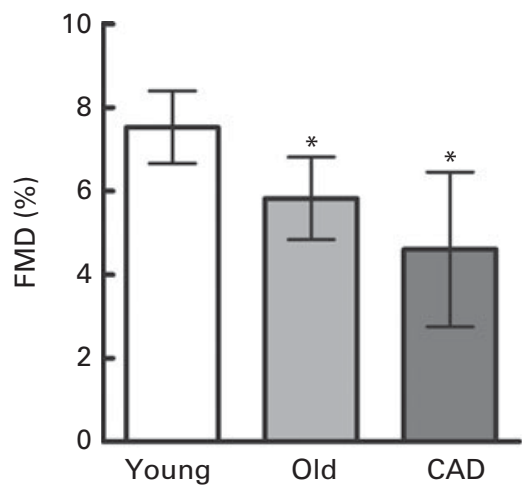

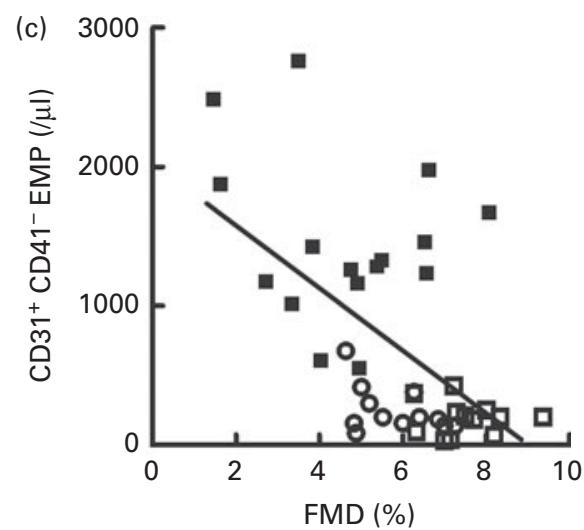

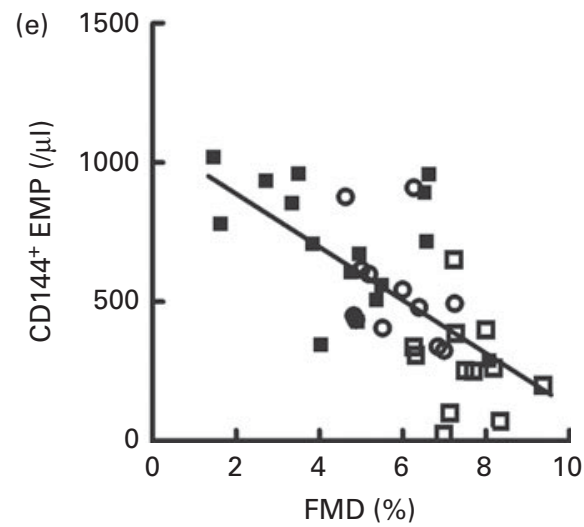

Fig. 1. Comparison of the endothelial function and endothelial microparticles (EMP) levels of patients with coronary artery disease (CAD) with those of the young and age-matched healthy control subjects. (a) Impaired endothelial function (assessed by flow-mediated vasodilation (FMD)) in patients with CAD and age-matched control subjects. (b) Increased CD31 $/ 41^{-}$EMP levels in patients with CAD when compared with the levels in young and age-matched healthy control subjects. (d) Increased $\mathrm{CD}_{144^{+}}$EMP levels in patients with CAD and age-matched control subjects when compared with the levels in young healthy control subjects. (c) and (e) levels of CD31 ${ }^{+} / 41^{-}$EMP $(r-0.59 ; P=0.01)$ and CD144 ${ }^{+}$EMP $(r-0.67 ; P=0.01)$ correlate with endothelial function as measured by FMD. ${ }^{\star}$ Mean values were significantly different from those of young healthy subjects $(P<0.05)$. † Mean value was significantly different from that of old healthy subjects $(P<0.05)$. $\square$, Young; $\bigcirc$, old; $\square, C A D$. 
$5.4 \mathrm{~mm}-\mathrm{KCl}$ and $6 \mathrm{mg} / \mathrm{ml}$ bovine serum albumin). After $15 \mathrm{~min}$ of incubation at $37^{\circ} \mathrm{C}, 25 \mu \mathrm{l}$ of the chromogenic substrate of Factor $\mathrm{Xa}$ Biophen CS-11(32) (Hyphen Biomedica; final concentration $0.44 \mathrm{mmol} / \mathrm{l}$ ) were added to each well of a ninety-six-well microtitre plate. The reaction was stopped immediately by the addition of $25 \mu \mathrm{l}$ of $50 \%$ (v/v) EDTA. Kinetic changes in absorbance at $405 \mathrm{~nm}$ were read on an ELISA reader at $37^{\circ} \mathrm{C}$. A standard curve was constructed using known concentrations of Factor Xa (Kordia) incubated under the same conditions with the chromogenic substrate.

\section{Statistical analysis}

The Kolmogorov-Smirnov test was used to confirm the normal distribution of EMP levels. Correlation was assessed by Pearson's coefficient. The primary test for an effect of the intervention was a test of interaction in a two-way repeatedmeasures ANOVA with Bonferroni post hoc test. Furthermore, we carried out a linear mixed model analysis to assess the interaction between period and intervention (carry-over) implementing the model proposed above with the subject as a random factor using the lmer() function from the lme 4 library in R. Data reported in the Results section are presented as means and standard deviations. Patients' characteristics, FMD and MP levels of the healthy subjects and CAD patients were analysed using one-way ANOVA with consecutive post hoc test. $P$ values $<0.05$ were considered to be statistically significant.

\section{Results}

Comparison of endothelial function and microparticle levels in patients with coronary artery disease and young and age-matched healthy subjects

Indicative of endothelial dysfunction, the CAD patients exhibited impaired flow-mediated vasodilation when compared with the twelve healthy young and twelve age-matched control subjects (4.6 (SD 0.8) v. 7.5 (SD 0.9)\% (young subjects: $P=0.01$ ) and $5 \cdot 8$ (sD 1.0) \% (age-matched subjects: $P=0 \cdot 01$ ) (Fig. 1(a))). At baseline, the levels of $\mathrm{CD} 31^{+} / 41^{-}$EMP ( $n$ 12) in the CAD patients were higher (1509 (SD 523) per $\mu \mathrm{l}$ ) than those in the age-matched (254 (SD 183) per $\mu l, P=0 \cdot 01$ ) and young healthy (196 (SD 124) per $\mu \mathrm{l}, P=0 \cdot 01$ ) control subjects (Fig. 1(b)). The levels of CD144 ${ }^{+}$EMP were higher in the CAD patients (707 (SD 174) per $\mu l, P=0.01$ ) and age-matched healthy control subjects (540 (SD 205) per $\mu l, P=0.02$ ) than in the young healthy control subjects (270 (SD 169) per $\mu \mathrm{l}$ ) (Fig. 1(d)). Overall, FMD was inversely correlated with the levels of $\mathrm{CD}_{3} 1^{+} / 41^{-}$and CD144 ${ }^{+}$EMP (Fig. 1(c) and (e); $r-0.67, P=0.01$ and $r-0.59, P=0.01$, respectively).
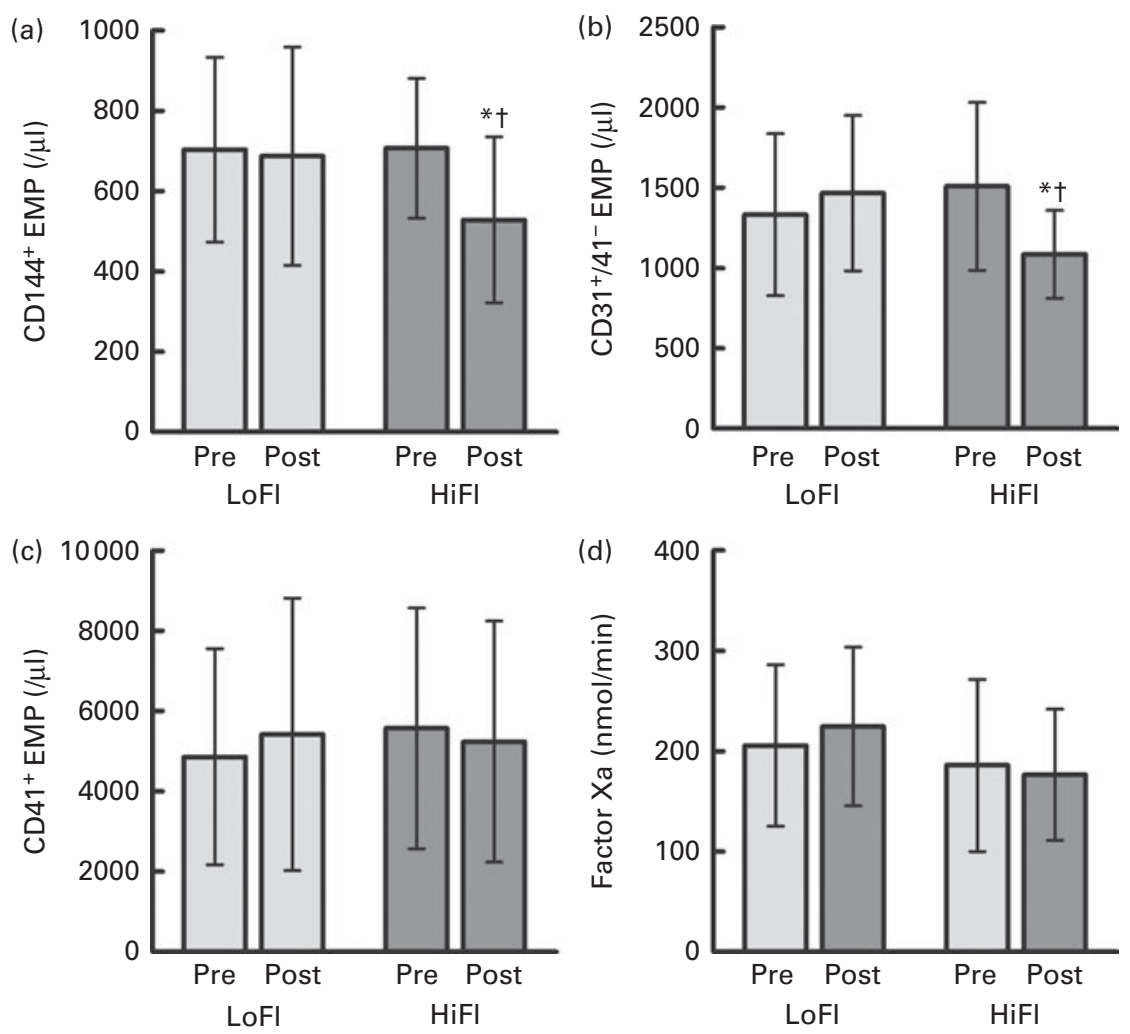

Fig. 2. High-flavanol intervention $(\mathrm{HiFI})$ decreased the levels of CD144 ${ }^{+}$endothelial microparticles (EMP) (a) and CD31 ${ }^{+} / 41^{-} \mathrm{EMP}(\mathrm{b})$ in the coronary artery disease patients but did not change the number of CD41 $1^{+}$platelet-derived microparticles (PMP) (c) or procoagulant activity of microparticles (MP) (d). Number of MP measured and coagulation activity of total MP measured as the formation of activated Factor $X$ per time before (pre) and after (post) 1 month of ingestion of high-CF drinks and low-flavanol drinks $\left(\mathrm{HiFI}\right.$ and $\mathrm{LoFI}$, respectively). ${ }^{*}$ Mean values were significantly different from those of respective baseline $(P<0.05)$. $†$ Mean values were significantly different from those of $\operatorname{LoFI}(P<0.05)$. 
High-flavanol intervention lowers the levels of endothelial microparticles in patients with coronary artery disease along with flow-mediated dilation improvement

In the present study, we demonstrated that in CAD patients together with the improvement of FMD, which we have shown previously ${ }^{(21)}$, the levels of circulating $\mathrm{CD} 144^{+}$EMP were significantly lowered from 707 (SD 174) to 528 (SD 207) per $\mu \mathrm{l}(-25.3 \%, P=0.01)$ after HiFI compared with baseline values before the initiation of the intervention, but not after LoFl (703 (SD 230) and 687 (SD 272) per $\mu l, P=1 \cdot 0$ ) (Fig. 2(a)). Furthermore, the number of $\mathrm{CD} 1^{+} / 41^{-}$EMP decreased from 1509 (SD 523) to 1086 (sD 274) per $\mu \mathrm{l}$ $(-23 \%, P=0.02)$ following HiFI, but not after LoFl (1332 (SD 504) to 1467 (SD 484) per $\mu l, P=1.00$ ) (Fig. 2(b)). In the CAD patients, the levels of $\mathrm{CD}_{144^{+}}$and $\mathrm{CD} 31^{+} / 41^{-} \mathrm{EMP}$ were inversely correlated with FMD $(r-0.49, P=0.01$ and $r-0.31, P=0.013$, respectively), suggesting that the number of EMP had decreased along with improvements in endothelial function. We did not observe any statistically significant interaction between period and intervention, suggesting the absence of a significant carry-over effect.

\section{High-flavanol intervention did not affect platelet-derived microparticles levels and microparticles-related procoagulant activity}

To test whether the effects observed were specifically related to endothelial cells or extended to other non-endothelial MP populations or biological functions of MP, we measured the levels of platelet-derived MP and the procoagulant activity of MP ex vivo. The levels of platelet-derived MP were affected neither by treatment with LoFl (4857 (SD 2688) v. 5418 (SD 3393) per $\mu \mathrm{l}, P=1 \cdot 0)$ nor by treatment with HiFI (5571 (SD 2996) v. 5236 (sD 3001) per $\mu l, P=1.0$ ) (Fig. 2(c)). Furthermore, the coagulation activity of total MP measured as the formation of activated Factor $\mathrm{X}$ per time did not significantly change after HiFI (186 (SD 86) v. 177 (SD 65) nM/min, $P=1 \cdot 0$ ) and LoFl (206 (sD 80) v. 225 (sD 79) $\mathrm{nm} / \mathrm{min}, P=1 \cdot 0$ ) (Fig. 2(d)).

\section{Discussion}

In the present study, in sixteen patients with CAD, we demonstrated that the levels of EMP were increased along with decreased endothelial function when compared with those of twelve age- and BMI-matched healthy control subjects and twelve young healthy subjects with low BMI. We have shown that a 1-month $\mathrm{CF}$ intervention improved endothelial function and enhanced the numbers of CAC in patients with $\mathrm{CAD}^{(21)}$. In the present study, we extend our finding by providing complementary evidence demonstrating that the improvement in endothelial function was paralleled by decreases in the levels of circulating EMP, a marker of endothelial integrity, towards control values.

Sabatier et $a l .{ }^{(23)}$ have proposed the concept of 'vascular competence'. The authors suggested that regenerative $v$. degenerative endothelial responses can be integrated in a clinical endothelial phenotype, reflecting the net result between damage from risk factors and endogenous repair capacity (i.e. EMP and CAC, respectively; Fig. 3). This endothelial phenotype characterises a vascular status that could define the 'vascular competence' of each individual. Indeed, one study has suggested that diet may be capable of improving endothelial phenotype in humans, thus re-establishing 'vascular competence ${ }^{(18)}$. In this randomised, cross-over dietary intervention study, the authors showed that consumption of Mediterranean diet, i.e. a diet rich in fruit and vegetables (which in turn is high in flavonoids), for 1 month can improve ischaemic reactive hyperaemia and that this is associated with a decrease in the levels of circulating EMP and increase in the number of CAC in healthy elderly subjects ${ }^{(18)}$. In the present study, similar responses were observed in elderly CAD patients after 1 month of flavanol intervention, suggesting that $\mathrm{CF}$ are capable of improving 'vascular competence'. Furthermore, we provide important contextual information as to how the EMP levels of CAD patients vary before and after intervention in relation to those of healthy controls. Our data suggest that flavanols decreased EMP values towards those of the healthy age-matched control subjects. However,

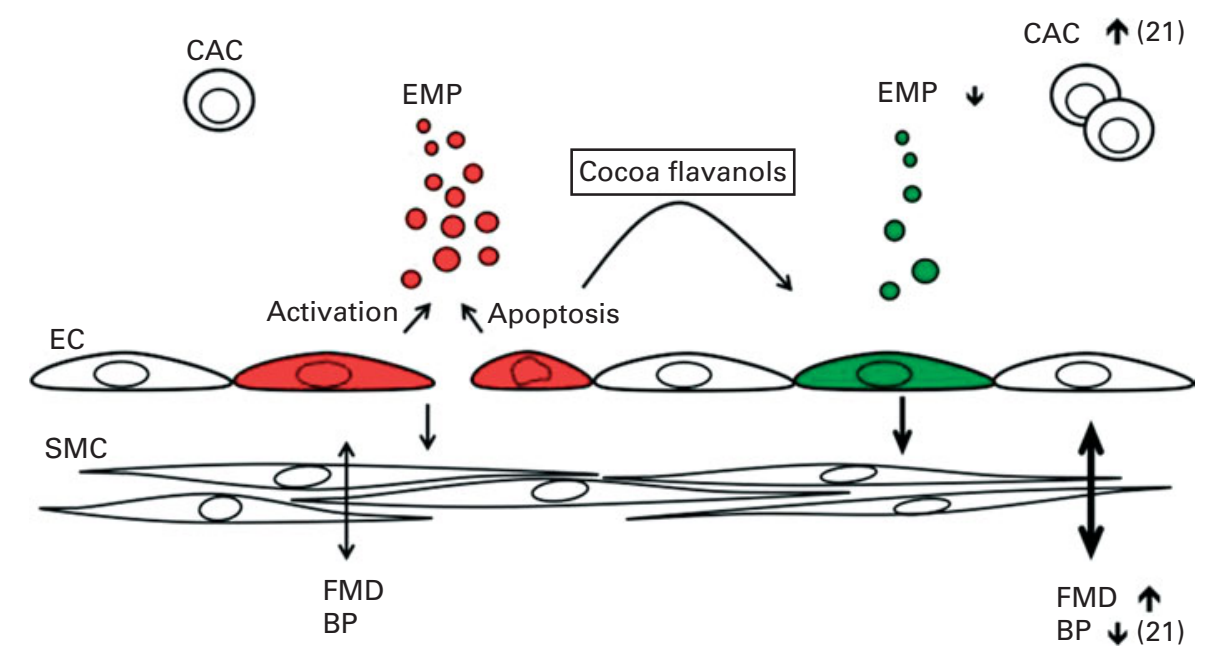

Fig. 3. Dietary flavanol intervention decreases the number of endothelial microparticles (EMP) in coronary artery disease patients. Improvement of endothelial integrity. EC, endothelial cells; SMC, smooth muscle cells; CAC, circulating angiogenic cells; FMD, flow-mediated vasodilation; BP, blood pressure. 
whether a long-term CF intervention will lead to a long-term clinical benefit remains to be shown.

The precise mechanisms leading to the generation of MP by endothelial cells has not been validated yet in vivo. The current knowledge on the formation of MP derives mainly from experiments on isolated or cultured cells, showing that both cell activation and cell apoptosis can lead to the release of MP by increasing intracellular Ca levels, decreasing membrane lipid asymmetry and reorganising cytoskeleton proteins $^{(24)}$. It is tenable to speculate that $\mathrm{CF}$ intake might prevent the release of MP by protecting endothelial cells via interaction with pathways leading to activation, apoptosis and survival, potentially involving enhanced NO bioavailability $^{(25,24)}$ and thereby improving endothelial function. However, in addition to being a marker of endothelial integrity, EMP have been shown to play an active role in the induction of endothelial dysfunction. Both in vivo- and in vitro-generated isolated EMP have been shown to impair endothelial function by decreasing the production of $\mathrm{NO}$ when incubated with rat aortic rings ex vivo ${ }^{(26,27)}$. Therefore, the decreased presence of EMP in circulation as observed in the present study might not only be a marker of improved vascular competence, but also directly contribute to the improvement of endothelial dysfunction.

Taken together, our data show that a flavanol intervention can lower the levels of EMP along with improvement in endothelial function, lending evidence to the concept that CF may be capable of improving endothelial integrity. These results provide novel insight into the potential mechanisms of flavanol bioactivities and underscore the potential of CF in the context of primary and secondary prevention.

\section{Acknowledgements}

The present study was supported by a grant from the American Heart Association to C. H. and Forschungskommission of the Medical Faculty, Heinrich-Heine University, to C. H. and an unrestricted research grant from Mars, Inc. to Y. Y., M. K. and C. H. Mars, Inc. provided the beverage powders for the preparation of the standardised test drinks used in the present study. C. H., H. S. and M. K. are senior investigators in the FLAVIOLA research consortium of the European Union (FP7-KBBE-2008-2B).

The authors' contributions are as follows: P. H. participated in study conception and design, data acquisition, and data analysis and interpretation; N. A. and F. S. A. aided in data analysis and interpretation, data acquisition, statistical analysis and technical support; R. S. aided in the acquisition and analysis of data and helped in reviewing the manuscript; M. K. aided in study supervision and administration and obtained funding; M. L. S. aided in the critical review of the manuscript for important intellectual content; Y. Y. provided material support and aided in the critical review of the manuscript; C. H. secured funding, aided in the conception and design of the study, analysis and interpretation of compiled data, drafting of the manuscript and critical revision of the manuscript for important intellectual content; B. S. aided in the interpretation and statistical analysis of data and drafting of the manuscript. All the authors were involved in the design of the present study.

H. S. is employed by Mars, Inc., and the company provided the beverage powders for the preparation of the standardised test drinks used in the present study. Y. Y., C. H. and M. K. have received unrestricted research grants from Mars, Inc.

\section{References}

1. Ross R (1993) The pathogenesis of atherosclerosis: a perspective for the 1990s. Nature 362, 801-809.

2. Woywodt A, Bahlmann FH, De Groot K, et al. (2002) Circulating endothelial cells: life, death, detachment and repair of the endothelial cell layer. Nephrol Dial Transplant 17, 1728-1730.

3. Martinez MC, Tesse A, Zobairi F, et al. (2005) Shed membrane microparticles from circulating and vascular cells in regulating vascular function. Am J Physiol Heart Circ Physiol 288, H1004-H1009.

4. Burnier L, Fontana P, Kwak BR, et al. (2009) Cell-derived microparticles in haemostasis and vascular medicine. Thromb Haemost 101, 439-451.

5. Rautou PE, Vion AC, Amabile N, et al. (2011) Microparticles, vascular function, and atherothrombosis. Circ Res 109, 593-606.

6. Horn P, Cortese-Krott MM, Amabile N, et al. (2012) Circulating microparticles carry a functional endothelial nitric oxide synthase that is decreased in patients with endothelial dysfunction. J Am Heart Assoc 2, e003764.

7. Werner N, Wassmann S, Ahlers P, et al. (2006) Circulating CD $31^{+}$/annexin $\mathrm{V}^{+}$apoptotic microparticles correlate with coronary endothelial function in patients with coronary artery disease. Arterioscler Thromb Vasc Biol 26, 112-116.

8. Sinning JM, Losch J, Walenta K, et al. (2011) Circulating CD $31^{+}$/annexin $\mathrm{V}^{+}$microparticles correlate with cardiovascular outcomes. Eur Heart J 32, 2034-2041.

9. Amabile N, Guerin AP, Tedgui A, et al. (2012) Predictive value of circulating endothelial microparticles for cardiovascular mortality in end-stage renal failure: a pilot study. Nephrol Dial Transplant 27, 1873-1880.

10. Lee ST, Chu K, Jung KH, et al. (2012) Circulating CD62E $\mathrm{E}^{+}$ microparticles and cardiovascular outcomes. PLOS ONE 7, e35713.

11. Stepien E, Stankiewicz E, Zalewski J, et al. (2012) Number of microparticles generated during acute myocardial infarction and stable angina correlates with platelet activation. Arch Med Res 43, 31-35.

12. Heiss C, Amabile N, Lee AC, et al. (2008) Brief secondhand smoke exposure depresses endothelial progenitor cells activity and endothelial function: sustained vascular injury and blunted nitric oxide production. J Am Coll Cardiol 51, $1760-1771$.

13. Navasiolava NM, Dignat-George F, Sabatier F, et al. (2010) Enforced physical inactivity increases endothelial microparticle levels in healthy volunteers. Am J Physiol Heart Circ Physiol 299, H248-H256.

14. Gordon C, Gudi K, Krause A, et al. (2011) Circulating endothelial microparticles as a measure of early lung destruction in cigarette smokers. Am J Respir Crit Care Med $\mathbf{1 8 4}$ $224-232$

15. Ferreira AC, Peter AA, Mendez AJ, et al. (2004) Postprandial hypertriglyceridemia increases circulating levels of endothelial cell microparticles. Circulation 110, 3599-3603.

16. Tushuizen ME, Nieuwland R, Rustemeijer C, et al. (2007) Elevated endothelial microparticles following consecutive 
meals are associated with vascular endothelial dysfunction in type 2 diabetes. Diabetes Care 30, 728-730.

17. Harrison M, Murphy RP, O'Connor PL, et al. (2009) The endothelial microparticle response to a high fat meal is not attenuated by prior exercise. Eur J Appl Physiol 106, 555-562.

18. Marin C, Ramirez R, Delgado-Lista J, et al. (2011) Mediterranean diet reduces endothelial damage and improves the regenerative capacity of endothelium. Am J Clin Nutr $\mathbf{9 3}$, 267-274

19. Heiss C, Keen CL \& Kelm M (2010) Flavanols and cardiovascular disease prevention. Eur Heart J 31, 2583-2592.

20. Gu L, Kelm MA, Hammerstone JF, et al. (2004) Concentrations of proanthocyanidins in common foods and estimations of normal consumption. J Nutr 134, 613-617.

21. Heiss C, Jahn S, Taylor M, et al. (2010) Improvement of endothelial function with dietary flavanols is associated with mobilization of circulating angiogenic cells in patients with coronary artery disease. J Am Coll Cardiol 56, 218-224.

22. Smith SC Jr, Allen J, Blair SN, et al. (2006) AHA/ACC guidelines for secondary prevention for patients with coronary and other atherosclerotic vascular disease: 2006 update: endorsed by the National Heart, Lung, and Blood Institute. Circulation 113, 2363-2372.

23. Sabatier F, Camoin-Jau L, Anfosso F, et al. (2009) Circulating endothelial cells, microparticles and progenitors: key players towards the definition of vascular competence. J Cell Mol Med 13, 454-471.

24. Skeppholm M, Mobarrez F, Malmqvist K, et al. (2012) Platelet-derived microparticles during and after acute coronary syndrome. Thromb Haemost 107, 1122-1129.

25. Heiss C, Schanz A, Amabile N, et al. (2010) Nitric oxide synthase expression and functional response to nitric oxide are both important modulators of circulating angiogenic cell response to angiogenic stimuli. Arterioscler Thromb Vasc Biol 30, 2212-2218

26. Boulanger CM, Scoazec A, Ebrahimian T, et al. (2001) Circulating microparticles from patients with myocardial infarction cause endothelial dysfunction. Circulation 104, 2649-2652.

27. Brodsky SV, Zhang F, Nasiletti A, et al. (2004) Endotheliumderived microparticles impair endothelial function in vitro. Am J Physiol Heart Circ Physiol 286, H1910-H1915. 